\title{
Expression of a marker for colonic crypt base cells is correlated with poor prognosis in human colorectal cancer
}

\author{
A A M van der Wurff, J ten Kate, P T J Marx, E P M van der Linden, C C L Beek, \\ F-J Bovelander, J Dekker, W N M Dinjens, M F von Meyenfeldt, J-W Arends, \\ F T Bosman
}

Department of Pathology, Maastricht University Hospital, Maastricht, The Netherlands

A A $M$ van der Wurff P T J Marx

E P M van der Linden

C C L Beek

J-W Arends

Department of Surgery, Maastricht University Hospital, The Netherlands $M \mathrm{~F}$ von Meyenfeldt

Department of Clinical Chemistry, De Wever

Hospital, Heerlen, The Netherlands

$\mathrm{J}$ ten Kate

Laboratory for

Pediatric

Gastroenterology and

Nutrition, Academic

Medical Centre,

Amsterdam, The

Netherlands

F-J Bovelander

J Dekker

Department of Pathology, Erasmus University, Rotterdam, The Netherlands W N M Dinjens

F T Bosman

Correspondence to: Dr van der Wurff,

Department of Pathology, St Elisabeth Hospital, PO Box 90151, 5000 LC Tilburg, The Netherlands.

Accepted for publication 16 July 1997

\begin{abstract}
Background-There is a need for markers in colorectal cancer which will allow subclassification of stage groups into subgroups with high versus low risk of recurrent disease.

Aims-To develop monoclonal antibodies that recognise antigens on immature crypt base cells, on the assumption that in a neoplasm undifferentiated but not the terminally differentiated cells will be responsible for tumour progression.

Methods-Colon crypt cells which were isolated from human colonic mucosa by EDTA/EGTA incubation were studied. By stepwise harvesting, crypt base cell enriched fractions were obtained, and after incubation with antibodies against dominant antigens, used as immunogens.

Results-Of one crypt base cell specific antibody (5E9), the reactive epitope appeared to be a non-terminal carbohydrate in the mucin O-glycans of the colon. The epitope did not seem to be colon specific, but was expressed in a variety of other tissues. In colorectal carcinomas, 5E9 immunoreactivity identified a subgroup of patients with a tendency for worse prognosis.
\end{abstract}

Conclusion-A mucin associated maturation epitope was identified in colonic crypt base cells, the expression of which in Dukes' stage B3 colorectal carcinoma may be associated with poor prognosis. (Gut 1998;42:63-70)

Keywords: colorectal adenocarcinoma; maturation epitope; stem cell; prognostic marker; mucin

In colorectal cancer, tumour stage as expressed in the Dukes' classification ${ }^{1}$ is still one of the best prognostic indicators. However, the behaviour of individual tumours within a stage is not uniform and therefore additional prognostic factors are needed for more accurate determination of tumour behaviour. One such prognostic indicator is histological grading ${ }^{2}$ which is, however, subjective and of limited significance.

In search for independent prognostic indicators, in addition to proliferation, oncogene status and ploidy, differentiation markers have been developed for the cell lineages in the colonic epithelium, which include columnar cells, mucin producing goblet cells, and neuroendocrine cells. In a large series of colorectal adenocarcinomas, markers for end stages of differentiation did not seem to have prognostic value. ${ }^{3}$ Only neuroendocrine markers correlated with worse prognosis ${ }^{4-6}$ which might reflect the relative immaturity of these cells, which are located basally in the crypt.

All colonic epithelial cells are thought to arise from a common progenitor cell, the stem cell, ${ }^{7-9}$ which is located at the bottom of the crypt and gives rise to daughter cells with proliferative capacity. During movement upwards in the direction of the lumen, the proliferative ability of the committed precursor cells for each of the three cell lineages is lost and gradual differentiation takes place. The fully differentiated cells are eventually shed into the bowel lumen.

For normal large bowel mucosa the stem cell concept appears valid and has gained wide acceptance. For carcinomas several observations support this concept. Firstly, in a small proportion of colorectal carcinomas a wide spectrum of differentiation has been found. ${ }^{10}{ }^{11}$ These findings are strongly in favour of the origin of these tumours from a multipotential stem cell and these "stem cell" carcinomas seem to have a particularly unfavourable prognosis. Secondly, in colon cancer cell lines induction of differentiation reduces the proliferation index. ${ }^{12-16}$ Thirdly, studies on human colon cancer cell lines have shown that these may exhibit a variable degree of proliferative activity, clonogenic potential, and cell differentiation. ${ }^{17-20}$ Finally, clonogenic assays generally show cloning efficiencies of tumour cell populations as low as $0.001 \%,{ }^{21}$ suggesting that the proliferating tumour cell fraction in human tumours is small.

Our working hypothesis is that in analogy with normal colonic mucosa, in colorectal cancer tumour stem cells exist. The proliferative capacity and stage of maturation of the tumour stem cells would be an important determinant of tumour behaviour: the more immature the tumour stem cell, the higher the proliferation index and the less differentiated and the more aggressive the tumour. To test the validity of this concept, we set out to generate monoclonal antibodies, which selectively stain crypt base cells, assuming that antigens in immature crypt base cells might also be expressed in colonic adenocarcinomas. In this paper, we describe the generation and characterisation, by way of immunohistochemistry, immunochemical, and biochemical analysis, of a new monoclonal 
antibody, 5E9, which selectively stains goblet cells in the lower half of the colonic crypt. The prognostic significance of its immunoreactivity was tested on a large series of colorectal adenocarcinomas. High expression of the 5E9 epitope appeared to be correlated with a tendency for poor prognosis in patients with Dukes' stage B3 cancer.

\section{Materials and methods}

MONOCLONAL ANTIBODY PRODUCTION

As a source of colon crypt epithelial cells, we used mucosa from neoplastic as well as non-neoplastic colectomy specimens. In specimens resected because of cancer, mucosa at a distance of at least $5 \mathrm{~cm}$ from the tumour was used. This was incubated, after decontamination with $0.04 \%$ (mass/volume) sodium hypochlorite in phosphate buffered saline (PBS) and $0.5 \mathrm{mmol} / 1$ dithiotreitol (DTT) in PBS to free the sample of mucus, with $0.05 \mathrm{mmol} / 1$ EDTA in calcium and magnesium free Hanks balanced salt solution (CMF-HBSS, Gibco, Paisley, Scotland) in a spinner flask (Gibco) according to ten Kate et al..$^{22}$ The mucosa with the remaining crypt bottom parts was incubated according to Whitehead $e t \mathrm{al}^{23}$ in a sterile petri dish containing $1 \mathrm{mmol} / \mathrm{l}$ EDTA (Merck, Darmstadt, Germany), $1 \mathrm{mmol} / 1$ ethyleneglycol-bis-( $\beta$-aminoethyl ether)N,N'-tetraacetic acid (EGTA, Sigma Chemical Company, St Louis, Missouri, USA), and 0.5 $\mathrm{mmol} / 1$ DTT (Sigma) in PBS ( $\mathrm{pH} 7.4$ ), for 30 minutes at room temperature. The crypts were liberated by vigorous shaking and harvested by centrifugation. The cells were lysed in buffer containing $5 \mathrm{mmol} / \mathrm{l} \mathrm{NaHPO}_{4}, \quad 1 \mathrm{mmol} / \mathrm{l}$ EDTA, $1 \mathrm{mmol} / 1 \beta$-mercaptoethanol, $0.125 \%$ (mass $/ \mathrm{mass}$ ) deoxycholate, $0.125 \%$ ( $\mathrm{vol} / \mathrm{vol}$ ) Triton X-100, and $250 \mathrm{mmol} / \mathrm{l}$ sucrose $(\mathrm{pH}$ 6.4) mixed with protease inhibitors $(1 \mathrm{mmol} / \mathrm{l}$ phenylmethylsulphonyl fluoride (Sigma), 2 mmol/1 N-ethylmaleimide (Sigma)).

To reduce the availability of major mucin antigens, ${ }^{24}$ the lysate containing the crypt bottom epithelium was incubated with antisera against colonic epithelium generated in mice and mixed with monoclonal antibodies against colonic mucins (Parlam 9 and 10). ${ }^{25}$ Bound antibodies were precipitated with Sepharose protein A (Pharmacia, Uppsala, Sweden) for one hour at $4^{\circ} \mathrm{C}$ ). Balb/c mice were immunised intrasplenally with $200 \mu \mathrm{l}$ of the final lysate, 72 hours prior to fusion. As repeated intrasplenal immunisations appeared not to be feasible, due to a high chance of rupture of the spleen, the mice were immunised only once. Spleen cells were fused with $\mathrm{Sp} \mathrm{2-0}$ myeloma cells and selected using standard procedures. ${ }^{26}$ Supernatant was tested on sections of normal colon and small bowel tissue specimens fixed in $70 \%$ alcohol as described by Dinjens et $a l^{27}$ to avoid epitope destruction by aldehyde fixation. Antibody producing wells were cloned and recloned by limiting dilution until all clones were productive. Isotyping was performed by immunohistochemistry on colonic epithelium using isotype specific secondary antibodies (Mouse typer, Biorad, Richmond). Specific positive and negative controls for each of the heavy and light chains were used.

IMMUNOREACTIVITY

Normal adult human tissues and tumours were retrieved from our files. Of each tissue, at least two specimens were tested and all tissues were obtained fresh, fixed in $4 \%$ (vol/vol) buffered formalin, dehydrated, and embedded in paraffin wax. Frozen tissue specimens were also used. Sections of $4 \mu \mathrm{m}$ thickness were cut and stained with an indirect immunoperoxidase technique. For antigen retrieval, sections were digested with proteases $\left(15\right.$ minutes at $37^{\circ} \mathrm{C}$ ) in concentrations ranging from $0.4 \%$ to $0.025 \%$ (mass/vol) for trypsin (in $0.4 \% \mathrm{CaCl}_{2}$, $\mathrm{pH} 7.8)$ and pepsin $(0.1 \mathrm{~mol} / 1 \mathrm{HCl})$. Pronase in $0.05 \mathrm{~mol} / 1$ Tris $\mathrm{HCl}(\mathrm{pH} 8)$ was also tested in concentrations of $0.04 \%$ to $0.0025 \%$ (mass/ vol) $\left(30\right.$ minutes at $\left.37^{\circ} \mathrm{C}\right)$. Immunoperoxidase staining was performed using rabbit antimouse IgG conjugated to horseradish peroxidase (HRP) as the detection system and diaminobenzidine (DAB) $-\mathrm{H}_{2} \mathrm{O}_{2}$ as the chromogenic substrate. For postembedding immunoelectron microscopy, $1 \mathrm{~mm}^{3}$ fragments of human colon mucosa taken from freshly obtained surgical specimens were fixed in $0.1 \mathrm{~mol} / 1$ phosphate buffer ( $\mathrm{pH} 7.2$ ) containing $1 \%$ (mass/vol) acroleine and $0.4 \%$ (mass/vol) glutaraldehyde at $4^{\circ} \mathrm{C}$ for four hours. Tissues were transferred and stored in a sucrose buffer of $1 \mathrm{~mol} / 1$ sucrose in $0.1 \mathrm{~mol} / 1$ phosphate buffer ( $\mathrm{pH} 7.2$ ) with $1 \%$ (vol/vol) paraformaldehyde at $4^{\circ} \mathrm{C}$ until further processing for standard Lowicryl embedding. ${ }^{28}$ After impregnation of the tissues with Lowicryl K4M (Baltzers, Liechtenstein) polymerisation was performed under ultraviolet exposure at $-35^{\circ} \mathrm{C}$. Ultrathin sections were incubated first with $10 \%$ (vol/vol) normal goat serum (NGS, Dakopatts, Glostrup, Denmark) in PBS (30 minutes at room temperature), second with the primary antibodies (one hour at room temperature), and finally with $10 \mathrm{~nm}$ colloidal gold labelled goat antimouse antiserum (GAM-10, Aureon, Wageningen, The Netherlands) under microwave irradiation at $150 \mathrm{~W}$ for 30 minutes. ${ }^{29}$ The immunogold-silver enhancement was done by magnification of the gold particles by precipitation of metallic silver (Aureon R Gent Developer and Enhancer, applied for two minutes at room temperature in the dark; Aureon). After rinsing in distilled water the sections were dried. The sections were contrasted with uranyl acetate and lead citrate. Micrographs were taken on a Zeiss EM 902 at $80 \mathrm{kV}$. Control sections were incubated with PBS. Background staining was always negligible.

BIOCHEMICAL CHARACTERISATION

A polyclonal antiserum was raised against human colonic mucin as described previously (anti-HCM). ${ }^{30}$ The characterisation of the mucin epitopes recognised by 5E9 was performed by western and spot blotting of purified colonic mucin, as described earlier for rat gastric mucin. ${ }^{31}$ Briefly, purified mucins were either run on $4 \%$ sodium dodecyl sulphate polyacrylamide gels and blotted onto nitrocellulose, or purified 
mucins were spotted directly on nitrocellulose using a vacuum operated spot blot apparatus (Biorad, Richmond, USA). Blots were incubated either with anti-HCM (for reasons of comparison) or spent 5E9 hybridoma culture medium, diluted $1 / 100$, followed by incubation with goat antirabbit IgG conjugated to HRP and stained with DAB. Specificity for peptide and carbohydrate epitopes was tested separately as follows:

(1) Proteinase K sensitivity prior to spot blotting (to determine the possible peptide nature of the epitope).

(2) Epitope sensitivity towards DTT reduction (to determine whether or not 5E9 detects conformational peptide epitope).

(3) Oxidative destruction of carbohydrate epitopes by periodic acid (to determine the carbohydrate nature).

(4) Incubation of 5E9 with the protease resistant glycopeptide of HCM (as an alternative method to show reactivity towards carbohydrate epitopes). To this end, HCM was digested for 24 hours with $10 \mu \mathrm{g} / \mathrm{ml}$ proteinase $\mathrm{K}$ (Merck) in $50 \mathrm{mM}$ Tris, $\mathrm{pH}$ 7.4 , at $37^{\circ} \mathrm{C}$. The proteinase $\mathrm{K}$ was inactivated by addition of an excess of phenylmethylsulphonyl fluoride (Sigma). The proteinase resistant glycopeptide was subsequently recovered in the void volume after Sephacryl S-200 (Pharmacia) gel filtration in $50 \mathrm{mM}$ Tris, $\mathrm{pH}$ 7.5. Isolated HCM glycopeptide was incubated for one hour at room temperature with the antibody prior to addition to the spot blotted mucin.

The recognition of mucin antigens was further tested in metabolic labelling experiments of colonic biopsy samples followed by immunoprecipitation of radiolabelled mucins, as described in detail elsewhere. ${ }^{30}$

To test whether the epitope recognised by $5 \mathrm{E} 9$ is a sialylated carbohydrate chain, neuraminidase digestion was performed according to Jass et $^{2} \mathrm{l}^{32} ; 1 \mathrm{mg}$ neuraminidase (Clostridium perfringens, Fluka 72202, Lot 326138/1) was dissolved in $0.425 \mathrm{ml}$ of $0.05 \mathrm{M}$ Tris $\mathrm{HCl}$ buffer, $\mathrm{pH} 7.0$, containing $0.01 \%$ (wt/vol) $\mathrm{CaCl}_{2}$. Saponification was achieved by immersing the slides in $0.5 \% \mathrm{KOH}$ in $70 \%$ ( $\mathrm{vol} / \mathrm{vol}$ ) alcohol (30 minutes at room temperature). Thereafter the slides were rinsed in $70 \%$ ( $\mathrm{vol} / \mathrm{vol}$ ) alcohol and washed in tap water for 10 minutes. As a positive control the lectin PNA (Boehringer Mannheim) was used with and without saponification.

STATISTICAL ANALYSIS

Colorectal adenocarcinomas of 350 patients were entered into two multicentre prospective clinical trials between 1979 and 1981. One trial was designed to compare patient survival after treatment of colonic cancer by conventional surgery or the no touch isolation technique. ${ }^{33}$ The other trial was conducted to compare survival in rectal cancer patients with or without preoperative radiotherapy. These patient series did not differ in overall survival; therefore, for the purpose of the present study, the patient groups were pooled. During the first three years follow up took place every three months; between three and five years after the initial diagnosis and surgery follow up was every six months. Standard protocols were conducted with routine blood counts and biochemistry studies (including carcinoembryonic antigen levels) at each visit and liver ultrasound, chest $x$ ray, and colonoscopy annually, to evaluate recurrence of disease and disease related death. After the initial five year follow up, during subsequent years only the time and cause of death were registered. In this study, failure was defined as death due to recurrent disease, excluding postoperative mortality within 30 days and non-related death. The correlation between monoclonal antibody 5E9 expression and other colorectal differentiation markers, Dukes' stage, differentiation grade, tumour size, localisation of the tumour within the large intestine, presence of (lymph) angioinvasion or perineural invasion, lymph node status, and central lymph node involvement was investigated in two way frequency tables using BMDP PC90 Statistical Software, program 4F (BMDP Statistical Software, Inc., Los Angeles, California). Interdependency of 5E9 expression and these variables was tested with Pearson's $\chi^{2}$ method. Scoring was done by two independent pathologists. 5E9 expression was compared with markers for the colorectal epithelial cell lineages. Mucin production was detected with the monoclonal antibody Parlam $3,{ }^{25}$ which detects a glycoprotein present in normal goblet cells and with the high iron diamine alcian blue (HID-AB) technique, which stains sulphated mucins brown. Columnar cells were identified by immunoreactivity for secretory component (SC). Neuroendocrine differentiation was detected with a monoclonal antibody against chromogranin A, the major component of neurosecretory granules.

As a clinicopathological staging system we used the Dukes' classification. ${ }^{1}$ Differentiation grade was assessed according to criteria outlined by Blenkinsopp et al. ${ }^{2}$ The prognostic value of $5 \mathrm{E} 9$ expression with respect to survival was investigated by univariate analysis, using Kaplan-Meier estimations and the stratified log rank test, with the program 4F, BMDP PC90 Statistical Software. To exclude the possibility of a type I error in the statistical analysis, a Bonferroni test was also performed.

\section{Results}

IMMUNOHISTOCHEMISTRY OF THE MONOCLONAL ANTIBODY 5E9

With the described immunisation protocol, the IgM $\kappa$ monoclonal antibody $5 \mathrm{E} 9$ was obtained, staining goblet cells located at the crypt base of normal colorectal epithelium (fig 1A). For immunohistochemical experiments at light microscopical level, immunostaining on standard paraffin wax embedded tissue with spent hybridoma supernatant was optimal in a dilution of $1 / 50$ in PBS- $1 \%$ bovine serum albumin (BSA), after enzyme digestion of the tissue sections with $0.01 \%$ pepsin in $0.1 \mathrm{~mol} / 1$ $\mathrm{HCl}\left(15\right.$ minutes at $\left.37^{\circ} \mathrm{C}\right)$. Comparison of unfixed cryostat sections with paraffin wax 

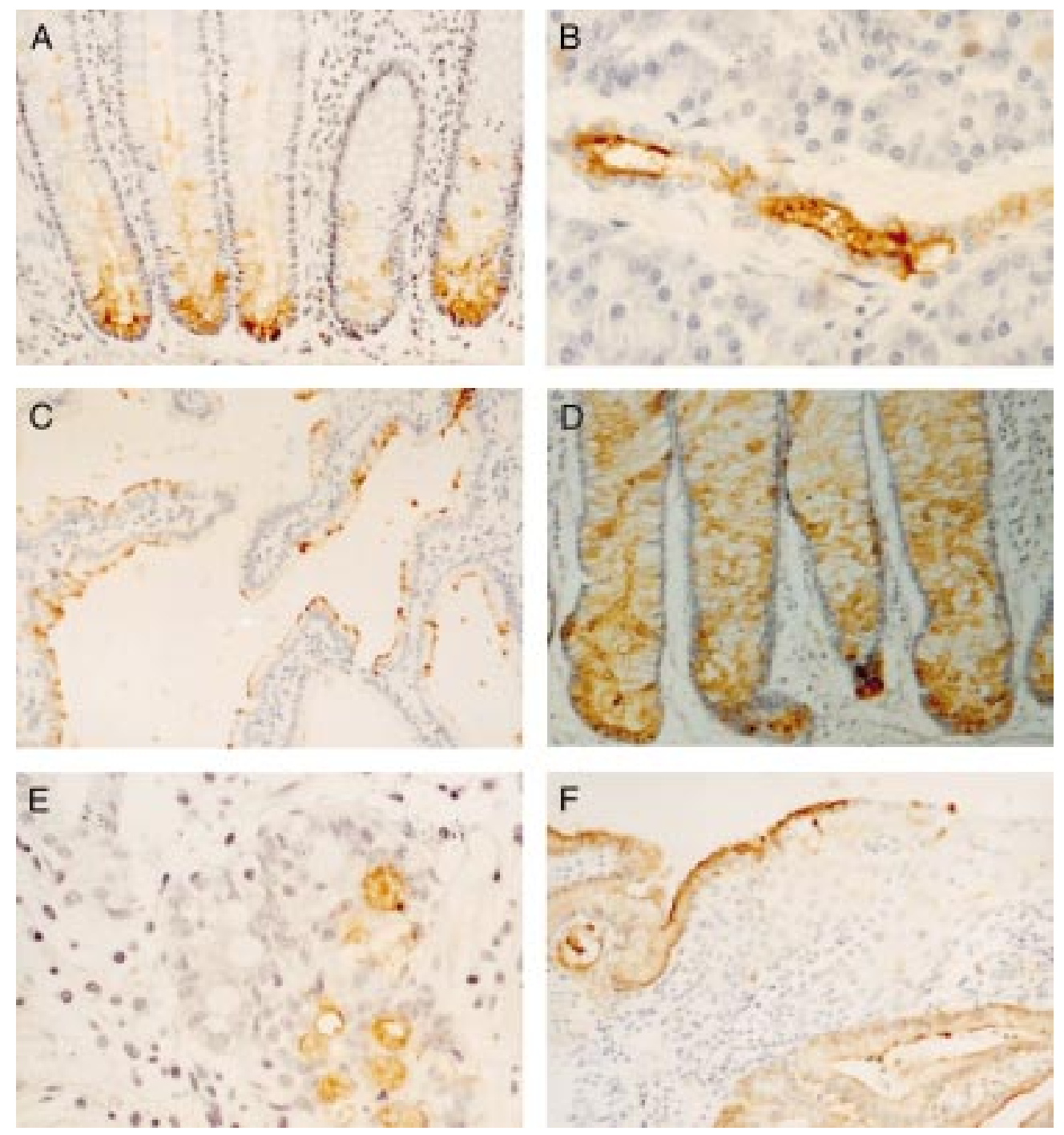

Figure 1 Immunoreactivity pattern of the monoclonal antibody $5 E 9$ on various normal human tissues: (A) normal colonic mucosa; (B) pancreas; (C) gall bladder; (D) transitional colonic mucosa; (E) intestinal metaplasia in the stomach; (F) cervix uteri: squamocolumnar junction.

sections after pepsin treatment showed an identical staining pattern for both conditions. Figures 1 and 2 show the immunoreactivity pattern of the monoclonal antibody 5E9 in normal and malignant human tissues. Normal mucosa from neoplastic as well as nonneoplastic colectomy specimens (proximal and distal colon) was used.

To exclude the possibility that 5E9 recognises a major blood group antigen we stained colon mucosa samples from patients with different $\mathrm{ABO}$ blood groups. In all groups an identical staining pattern was obtained. In the digestive tract, immunoreactivity was noted in submucosal glands of the oesophagus, duct epithelium of the parotid gland, goblet cells at the lower half of the crypt in the duodenum, some pancreatic duct cells (fig 1B), and in the mucosa lining the gall bladder (fig lC). Pancreatic islets were not stained. In normal colonic mucosa, from neoplastic as well as nonneoplastic resection specimens, only crypt base cells showed 5E9 immunoreactivity. Strikingly, immunoreactivity was observed in all goblet cells in colonic epithelium adjacent to adeno- carcinomas (transitional mucosa, fig 1D). In the stomach, reactivity was noted only in some goblet cells in areas of intestinal metaplasia (fig $1 \mathrm{E})$. In the urogenital tract, staining was observed in endometrial and endocervical glandular epithelium (fig 1F). Furthermore, reactivity was found in some acinar and duct cells of the breast. Focal reactivity was also observed in prostate and bladder epithelium. In normal ovary tissue, no immunoreactivity was found. In cystadenomas and adenocarcinomas of the ovary, however, whether serous or mucinous, focal immunoreactivity was noted. In the respiratory tract, bronchial epithelium, pneumocytes type II and few goblet cells in the lining tracheal epithelium showed staining. Carcinomas in urogenital and respiratory tract showed mostly a focal immunoreactivity pattern.

Colorectal adenocarcinomas showed 5E9 immunoreactivity in $33 \%$ of our 297 cases. The proportion of positive cases did not differ appreciably for different periods of collection of patient material, which makes it unlikely that variations in tissue preparation techniques or 

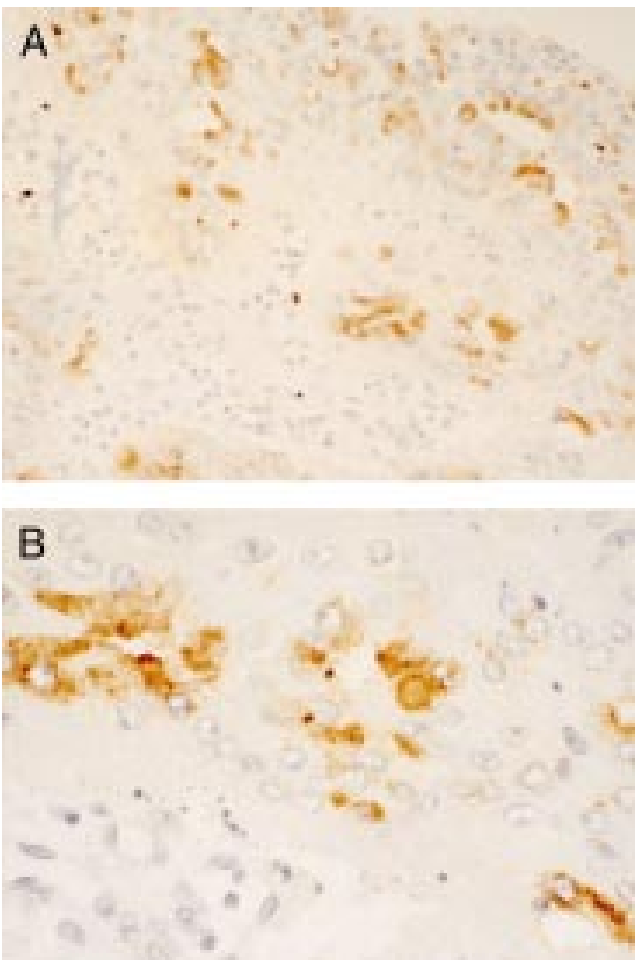

Figure 2 5E9 immunoreactivity pattern in colorectal adenocarcinoma: $(A)$ overview; (B) detail.

limited antigen stability might have influenced the immunohistochemical results. The staining was often intracellular, but also apical membrane associated; some single cells were frequently homogeneously stained, but occasionally diffuse staining was observed (fig 2). By immunoelectron microscopy the reactivity was located intracellularly on the outer rim of mucin vacuoles (fig 3). Staining of the Golgi complex was not observed.

\section{BIOCHEMICAL CHARACTERISATION OF THE 5E9} EPITOPE

As colonic mucosa was used as antigen source, mucins seemed to be the likely candidates for the antigen recognised by 5E9. Therefore, studies were undertaken to define this antigen, using purified HCM for comparison. Previous studies had shown that anti-HCM specifically recognises polypeptide epitopes of Muc2. ${ }^{30}$ On western blotting, HCM was recognised by both anti-HCM and $5 \mathrm{E} 9$ as a $550 \mathrm{kDa}$ band. This band was previously identified as human colonic Muc2, the major secretory mucin in the colon, which is produced in the colonic goblet cells. ${ }^{30}$ Digestion with proteinase $\mathrm{K}$ resulted in a decrease in the immunoreactivity of 5E9 towards HCM, while reduction by DDT had no effect. Oxidation of carbohydrate epitopes by periodic acid resulted in complete loss of reactivity. Furthermore, preincubation of 5E9 with the HCM glycopeptide, isolated after extensive proteinase $\mathrm{K}$ digestion, inhibited the binding of 5E9 with spot blotted HCM. The reactivity of anti-HCM towards HCM, which reacts primarily with peptide epitopes, showed a converse reactivity compared with 5E9: proteinase $\mathrm{K}$ destroyed all reactivity, while oxidation by periodic acid and preincubation with HCM glycopeptide had no effect on reactivity. These results indicate that 5E9 most likely recognises a carbohydrate epitope of HCM. In metabolic labelling studies using $5 \mathrm{E} 9$, we were unable to immunoprecipitate ${ }^{35} \mathrm{~S}$ sulphate labelled mature Muc2, or its ${ }^{35} \mathrm{~S}$ amino acid labelled precursor. As a positive control, immunoprecipitations from the same tissue homogenates, using anti-HCM, yielded the expected $600 \mathrm{kDa}{ }^{35} \mathrm{~S}$ amino acid labelled Muc2 precursor, and the $550 \mathrm{kDa}^{35} \mathrm{~S}$ sulphate labelled mature Muc2.

5E9 immunoreactivity could not be abolished by neuraminidase digestion with or without prior saponification. Lectin PNA, used as a positive control, showed the expected reactivity pattern.

CLINICOPATHOLOGICAL ANALYSIS

The 350 patients in this study were 165 men and 185 women, ranging in age from 29 to 91 years (median age 69 years). 5E9 staining was performed on available tissue specimens of 297 patients (192 colonic adenocarcinomas and 105 rectal carcinomas). These were comparable in Dukes' stage and histological grading to the overall population. To obtain subgroups suitable for statistical analysis, the subgroups were combined: group A tumours consisted of colorectal adenocarcinomas of Dukes' stage A, B1, and B2; group B consisted of Dukes' stage B3; group C combined Dukes' stage C1 and C2 tumours; and group D contained Dukes' stage $\mathrm{D}$. Of the tumours, using the above mentioned Dukes' combinations, 63 were group A, 117 group B, 91 group C, and 26 group $\mathrm{D}$. The number of well differentiated tumours was 31 ; 227 tumours were moderately differentiated and 32 poorly differentiated (seven tumours
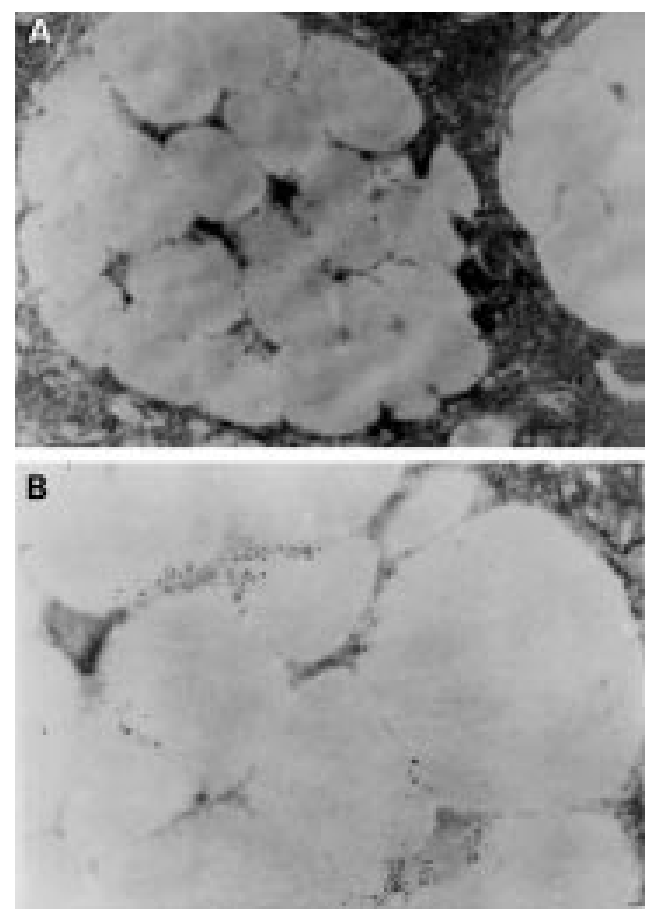

Figure 35 E9 immunoelectron microscopy. 5E9 immunogold reactivity is located intracellularly in goblet cells on the outer rim of mucin vacuoles: $(A)$ overview; $(B)$ detail. 

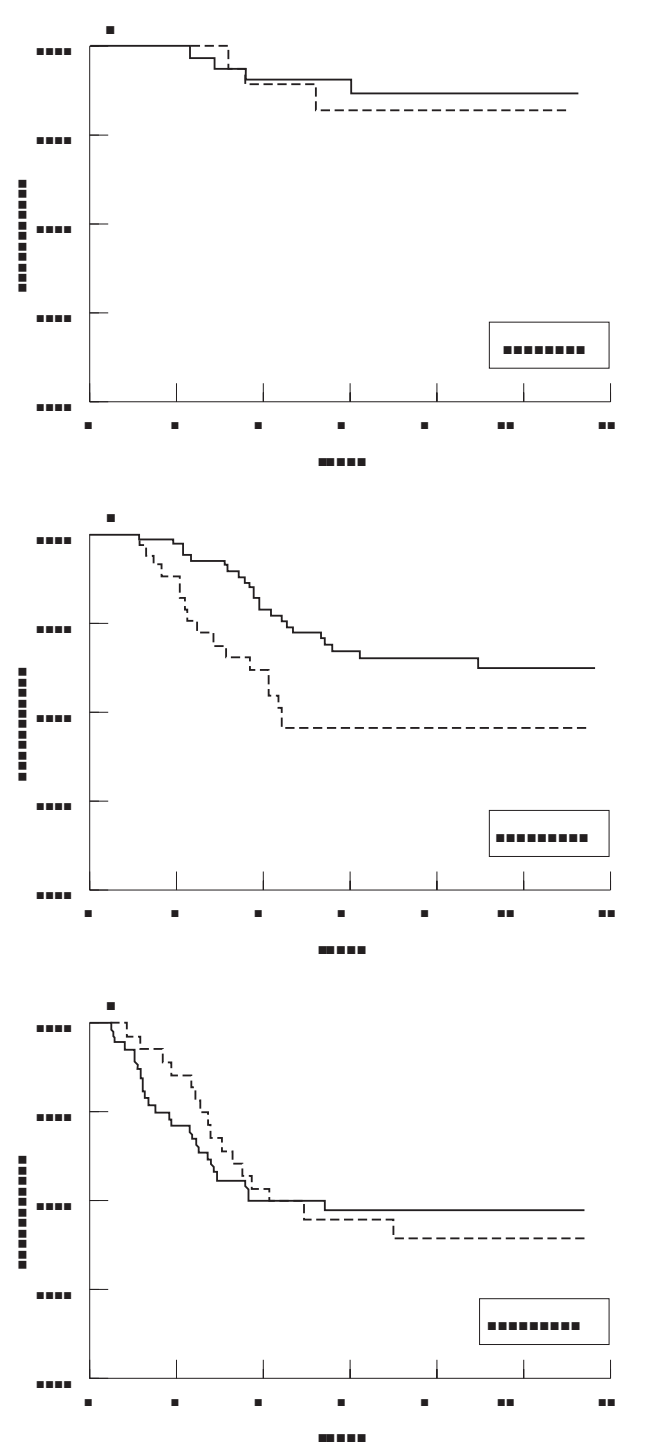

Figure 4 Kaplan-Meier survival curves for colorectal adenocarcinoma with 5E9 immunoreactivity: (A) Dukes' stage $A, B 1$, and $B 2(p=0.75, n=63)$; (B) Dukes'stage B3 $(p=0.037, n=117) ;(C)$ Dukes' stage $C 1$ and $C 2(p=0.95$; $n=91)$. Dotted lines, $5 E 9$ expression; solid lines, no $5 E 9$ expression.

were not graded). 5E9 positive cells were found in 97 tissue specimens of colorectal cancer. Multiple two way frequency tables were prepared yielding two positive results, namely with chromogranin $\mathrm{A}$ and with HID-AB expression. There seems to be a trend towards a positive correlation of $5 \mathrm{E} 9$ expression with neuroendocrine differentiation $(\mathrm{p}=0.01)$ as well as with HID-AB, a sulphomucin stain $(p=0.04)$. Figure 4 presents Kaplan-Meier survival analysis for the patient subgroups. A stratified log rank test was used, which showed non-significance $(p=0.265)$. If however the different Dukes' groups are taken into account, $5 E 9$ immunoreactivity was noted in $23,39,31$, and 11 cases in groups $\mathrm{A}, \mathrm{B}, \mathrm{C}$, and $\mathrm{D}$, respectively. It appeared that in group B (Dukes' stage B3) 5E9 expression was positively correlated with worse prognosis $(p=0.037)$. In the Bonferroni test, to exclude a type I error, this result was not sustained.

\section{Discussion}

The histological classification of colorectal adenocarcinoma has not changed in recent years. In addition to adenocarcinomas, mucinous, signet ring, and undifferentiated carcinomas are distinguished (World Health Organisation). Furthermore, grading is performed in a three grade system, ${ }^{2}$ which falls short in reproducibility and has limited prognostic significance. Various attempts have been made to improve classification using markers for end differentiational stages of the cell lineages in colonic epithelium. ${ }^{3-6}$ Secretory component (SC) is a marker for enterocytes, lysozyme is a marker for Paneth cells, different mucin markers identify goblet cells, and neuroendocrine markers identify neuroendocrine cells. Mucins, SC, and lysozyme are often coexpressed in a large proportion of the adenocarcinomas and for these markers a consistent prognostic significance has not been reported. With regard to neuroendocrine markers, expression of chromogranin A was repeatedly found to correlate significantly with worse prognosis. ${ }^{56}$

In looking for new markers for classification of colorectal cancer we reasoned that the behaviour of the tumour is largely determined by the proliferating stem cell fraction and not by terminally differentiated cells. Therefore, it would be plausible to look for markers for the undifferentiated (stem) cell fraction of the tumour. In the search for such markers we reasoned that crypt base cell associated antigens might also identify immature tumour (stem) cells.

Using as an antigen source epithelium of crypt bases, which was incubated with antibodies against major mucin epitopes and with antisera against colonic epithelium generated in mice to block for dominant epitopes, we succeeded in developing an antibody against a subset of goblet cells located in the lower half of the crypt, around and just above the proliferation zone. The epitope of this monoclonal antibody is expressed in a diversity of human tissues, both normal and malignant, but shows a very specific staining pattern in the colon. Immunoelectron microscopy showed reactivity in the outer rim of mucin vacuoles in goblet cells. It is of interest that in the digestive tract 5E9 staining was found in goblet cells in intestinal metaplasia in gastric mucosa as well as in Barrett's oesophagus. Both conditions carry an increased risk for the development of adenocarcinoma. In contrast to staining of basally located goblet cells in normal colonic mucosa, all goblet cells were stained in transitional mucosa neighbouring adenocarcinoma. Ultrastructural studies have indicated that crypt epithelial cells in transitional mucosa show slower maturation than normal. ${ }^{34}$ The increase of 5E9 positive cells in transitional mucosa could be the result of such a delay in the process of crypt cell maturation and this observation would be in keeping with the lower differentiation level of 5E9 expressing goblet cells.

In the search for the epitope recognised by $5 \mathrm{E} 9$, immunoblotting showed that $5 \mathrm{E} 9$ recognises an epitope present on an HCM associated glycoprotein, identified as human colonic 
Muc2. ${ }^{30}$ The blocking of 5E9 staining by periodic acid oxidation and by preincubation with the HCM glycopeptide indicates that 5E9 recognises a carbohydrate (O-glycans) epitope on Muc2. Although the monoclonal antibody recognised Muc2 in blots, it was not able to retrieve the antigen by immunoprecipitation from homogenates. Possible explanations could he scarcity of the O-glycan epitope, the absence of an O-glycan in Muc2, ${ }^{35}$ or low affinity of 5E9, which is not entirely unlikely given the $\operatorname{IgM}$ isotype of the antibody. 5E9 specifically stained the mucin granules in goblet cells low in the colonic and small intestinal crypts of all individuals tested, indicating that the carbohydrate epitope was not a blood group antigen or an antigen covered by blood group saccharides. The crypt base specificity of 5E9 staining suggests that the Muc2 epitope, recognised by $5 \mathrm{E} 9$, becomes masked when the goblet cells differentiate further during their migration upward from the bottom of the crypt. This could imply that the O-glycosylation pattern of Muc2 changes when the crypt cells migrate upward. The epitope recognised by $5 \mathrm{E} 9$ seems not to be sialic acid given the insensitivity to neuraminidase treatment with or without prior saponification. In the literature other monoclonal antibodies such as SIMA ${ }^{36}$ and $\mathrm{Stn},{ }^{32}$ with a comparable immunoreactivity pattern, are described. These antibodies recognise sialylated carbohydrate chains.

To evaluate the potential prognostic significance of 5E9 expression in colorectal adenocarcinomas, we stained a series of human colorectal carcinomas and compared the 5E9 status with various earlier studied pathological variables. We noted a trend towards 5E9 expression along with chromogranin A defined neuroendocrine and HID-AB defined sulphomucin expression. Both characteristics were earlier found to be associated with worse prognosis. ${ }^{56}$ The stratified log rank test did not yield any significance. In our group A (Dukes' stage $\mathrm{A} 1, \mathrm{~B} 1$, and $\mathrm{B} 2$ ) and group $\mathrm{C}$ carcinomas (Dukes' stage C1 and C2), 5E9 staining did not have any prognostic significance. In Dukes' stage B3 patients, however, a group of patients with a heterogeneous chance of survival, 5E9 appeared to be a positive prognostic variable, identifying a subgroup of patients with a worse prognosis. This increased expression of the 5E9 epitope in prognostically unfavourable Dukes' stage B3 adenocarcinomas can indicate that a change in the O-glycosylation of Muc2 in colonic tumour cells might take place during progression towards recurrent (incurable) disease.

In conclusion, we have developed a new monoclonal antibody which most likely recognises a non-terminal carbohydrate epitope on the Muc2 O-glycans in the colon and which reacts specifically with crypt base cells in colonic mucosa. In group B, Dukes' stage B3 adenocarcinomas, 5E9 staining identifies a subset of tumours with a tendency for worse prognosis. Studies on more cases are needed to show whether these results prove to be statistically significant. Also, the clinical value of 5E9 for classifying colorectal cancer needs to be evaluated further.

W N M Dinjens and F T Bosman were supported by grant EUR-90-53 from the Dutch Cancer Society. The skilful contribution of A de Jong in the immunoelectron microscopy experiments is gratefully acknowledged. Thanks are due to A P de
Bruine for fruitful discussions and to H A J Bruinsma for additional immunohistochemistry.

1 Dukes CE. The classification of cancer of the rectum. $\mathcal{F}$ Pathol Bacteriol 1932;35:323-32.

2 Blenkinsopp WK, Stewart-Browns, Blesovsky L, et al. Histopathology reporting in large bowel cancer. f Clin Pathol 1981:34:598-613.

3 Ho SB, Itzkowitz SH, Friera AM, et al. Cell lineage markers in premalignant and malignant colonic mucosa. Gastroenterology 1989;97:392-404.

4 Arends SW, Wiggers T, Verstijnen K, Bosman FT. The occurrence and clinicopathological significance of serotonin immunoreactive cells in large bowel carcinoma. $\mathcal{f}$ Pathol 1986;149:97-102.

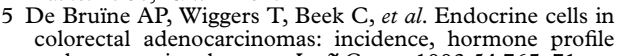
and prognostic relevance. Int $\mathcal{F}$ Cancer 1993;54:765-71.

6 Hamada Y, Oishi A, Shoji T, et al. Endocrine cells and prognosis in patients with colorectal carcinoma. Cancer 1992;69:2641-6.

7 Chang WW, Leblond CP. An unitarian theory of the origin of the three populations of epithelial cells in the mouse large intestine. Anat Rec 1971;169:293.

8 Griffiths DFR, Davies SJ, Williams GT, Williams ED. Demonstration of somatic mutation and colonic crypt
clonality by X-linked enzyme histochemistry. Nature 1988; 333:461-3.

9 Kirkland SC. Clonal origin of columnar, mucous, and endocrine cell lineages in human colorectal epithelium. Cancer 1988;61:1359-63.

10 Damjanov I, Amenta P, Bosman FT. Undifferentiated carcinoma of the colon containing exocrine, neuroendocrine and squamous cells. Virchows Arch 1983;401:57-66.

11 Novello P, Duvillard P, Grandjouan S, et al. Carcinomas of the colon with multidirectional differentiation. Dig Dis Sci 1995;40:100-6.

12 Meyer JS, Rao BS, Stevens SC, White WI. Low incidence of estrogen receptor in breast carcinomas with rapid rates of cellular replication. Cancer 1979;40:2290-8.

13 Turowski GA, Rashid Z, Hong F, Madri JA, Basson MD. Glutamine modulates phenotype and stimulates proliferation in human colon cancer cell lines. Cancer Res 1994;54: 5974-80.

14 Sakamoto K, Venkatraman G, Shamsuddin AM. Growth inhibition and differentiation of HT-29 cells in vitro by inositol hexaphosphate (phytic acid). Carcinogenesis 1993; 14:1815-9.

15 Zhao X, Feldman D. Regulation of vitamin D receptor abundance and responsiveness during differentiation of HT-29 human colon cancer cells. Endocrinology 1993;132: 1808-14.

16 Baghdiguian S, Verrier B, Gerard C, Fantini J. Insulin like growth factor $I$ is an autocrine regulator of human colon cancer cell differentiation and growth. Cancer Lett 1992;62: 23-33.

17 Mackillop WJ, Stewart SS, Buick RU. Density/volume analysis in the study of cellular heterogeneity in human analysia in the study of cellular heterogeneity

18 Bizzari JP, Mackillop WJ, Buick RN. Cellular specificity of NB70K, a putative human ovarian antigen. Cancer Res 1983;43:864-7

19 Itzkowitz SH, Shi ZR, Kim YS. Heterogeneous expression of two oncodevelopmental antigens, CEA and SSEA-1, in colorectal cancer. Histochem $\mathcal{F}$ 1986;18:155-63.

20 Brattain MG, Levine AE, Chakrabarty S, Yeoman LC. Hetrogeneity of human colon carcinoma. Cancer Metastasis Rev 1984;3:177-91.

21 Salmon SE. Human tumor colony assay and chemosensitivity testing. Cancer Treat Rep 1984;68:117-25.

22 ten Kate J, Verspaget $\mathrm{H}$, Wijnen J, et al. Maturation dependent changes in nucleoside phosphorylase (NP), adenosine deaminase (ADA) and ADA complexing protein (ADCP) in intestinal epithelial cells. In: Peeters H, ed. Pro(ADCP) in intestinal epithelial cells. In: Peeters H, ed. ProFluids. Oxford: Pergamon Press, 1985:339-42.

23 Whitehead RH, Brown A, Bhatal PS. A method for the isolation and culture of human colonic crypts in collagen gels. In Vitro Cell and Developmental Biology 1987;23:436-42.

24 King SW, Morrow KJ. Monoclonal antibodies produced against antigenic determinants present in complex mixtures of proteins. Biotechniques 1988;6:856-61.

25 Verstijnen CPHJ, Arends JW, Moerkerk PTM, et al. Colonic epithelium reactive monoclonal antibodies. Identification and immunohistochemical localization of the target epitopes. Histochemistry 1989;92:397-406.

26 Köhler G, Milstein C. Continuous cultures of fused cells secreting antibodies of predefined specificity. Nature 1975; 256:495-7.

27 Dinjens WNM, vd Linden EPM, Signet C, et al. Solid-phase adsorption of antigens for efficient production of antibodies reactive with native and fixed tissue antigens. F Immunol Methods 1990;126:175-82. 
28 Van der Kwast TH, Versnel MA, Delahaye M, et al. Expression of epithelial membrane antigen on malignant mesothelioma cells: an immunocytochemical and immunocl
tron microscopic study. Acta Cytol 1988;32:169-74.

29 Zondervan PE, De Jong A, Sorber CWJ, et al. Microwave stimulated incubation in immunoelectron microscopy: a quantitative study. Histochem $\mathcal{F}$ 1988;20:359-64.

30 Tytgat KMAJ, Büller HA, Opdam FJM, et al. Biosynthesis of human colonic mucin: Muc2 is the prominent secretory mucin. Gastroenterology 1994;107:1352-63.

31 Dekker J, Van Beurden-Lamers WMO, Strous GJ. Biosynthesis of rat gastric mucin. F Biol Chem 1989;264:10431-7.

32 Jass JR, Allison LJ, Edgar SG. Distribution of sialosyl Tn and $\mathrm{Tn}$ antigens within normal and malignant colorectal epithelium. F Pathol 1995;176:143-9.
33 Wiggers T, Jeekel J, Arends JW, et al. No-touch isolation technique in colon cancer: a controlled prospective trial. $\mathrm{Br}$ f Surg 1988;75:409-15.

34 Riddel RH, Levin B. Ultrastructure of the transitional to large bowel carcinoma. Cancer 1977;40:2509-22.

35 Dekker J, Strous GJ. Covalent oligomerization of rat gastric mucin occurs in the rough endoplasmatic reticulum, is $\mathrm{N}$-glycosylation-dependent, and precedes initial O-gly$\mathrm{N}$-glycosylation-dependent, and precedes
cosylation. F Biol Chem 1990;30:18116-22.

36 Pilbrow SJ, Hertzog PJ, Pinczower GD, Linnane AW. Expression of a novel family of epitopes on small intestinal mucins in colorectal cancers, adjacent and remote mucosa. Tumor Biol 1992;13:251-67. 